\title{
A feasibility study on the adoption of e-learning for public health nurse continuing education in Taiwan
}

\author{
Shu Yu ${ }^{\text {a,b }}$, I-Ju Chen ${ }^{a}$, Kuei-Feng Yang ${ }^{a}$, Tze-Fang Wang ${ }^{\text {a }}$,Lee-Lan Yen ${ }^{\mathrm{b}, *}$ \\ a School of Nursing, National Yang-Ming University, No.155, Section 2, Li-Nong Street, Taipei 112, \\ Taiwan, ROC \\ b Institute of Health Policy and Management, National Taiwan University, Room 623 , No.17, \\ Hsu-Chow Road, Taipei 100, Taiwan, ROC
}

Accepted 23 October 2006

\section{KEYWORDS \\ Public health nurses; Continuing education; Web-based learning; E-learning}

\begin{abstract}
Summary The purpose of this study is to investigate the feasibility of developing elearning and to examine reasons for adopting or rejecting e-learning as an alternative way to conduct continuing education (CE) for public health nurses (PHNs). A nationwide-based cross-sectional study was conducted with a randomly selected sample of 233 PHNs in Taiwan. A structured questionnaire was used to collect data by mailing methods. The majority of PHNs $(88.84 \%, n=207)$ showed an affirmative intention towards adopting e-learning as their one way of CE. Reasons for adopting e-learning included achieving life learning, fulfilling personal interests, time-saving, based on job needs, information diversity, flexible in time and space, self-regulatory learning, cost-effectiveness, less impact on family duties and life. Twenty-six PHNs (11.16\%) who rejected e-learning as their way of CE indicated main reasons including poor computer competence, lack of personal computer and without internet access, heavy work load, heavy family duties, conflict with personal preference, heavy economic burden, lack of motivation, and low self-control. This study reveals a high feasibility of developing e-learning that coexists with other CE models (e.g. traditional instruction). Reasons analyses provide directions for decreasing barriers for developing a learning model of this new medium for nurses' CE. (c) 2006 Elsevier Ltd. All rights reserved.
\end{abstract}

\section{Introduction and literature review}

${ }^{*}$ Corresponding author. Tel.: +886 2 33228062; fax: +886 2 2391778.

E-mail address: lan@ha.mc.ntu.edu.tw (L.-L. Yen).
With rapid changes occurring in health care environments, it is critical for health care professionals to keep pace with updated information. The 
e-learning program (or web-based learning) involves learning activities, either on-line or off-line, which is carried out with digital tools and access to teaching materials through wired and wireless networks. Public health nurses (PHNs) are expected to play the gatekeepers of public health and, as such, constantly need to update their professional knowledge and skills to ensure the quality of health care (Gebbie, 1999). Similarly, in Taiwan, PHNs working in public health centers play major roles in community health care (Yu and Chin, 1996).

In an era of knowledge economy, the Internet, a relatively new medium, is expected to play an important part in providing continuing education (CE) for health professionals, i.e., PHNs in this study. The use of computers is widespread and increasing, especially with the advancement of software and hardware platforms. PHNs who need continuing education can now select the most suitable learning style for themselves (Billings et al., 2001; Wills and Stommel, 2002; Frith and Kee, 2003). E-learning, as a diverse learning style, has been widely recognized in several countries. E-learning has become a valuable and legitimate learning method for health care professionals in the 21st century (Cook et al., 2004; Leasure et al., 2000; Kearns et al., 2004; Fullerton and Ingle, 2003; Bernhardt et al., 2003; DiMaria-Ghalili et al., 2005). However, research in the application of web-based learning in providing CE for health professionals is still in its early stage. Although most nurses reveal a positive attitude towards e-learning (Yu and Yang, 2006), whether and why nurses, PHNs in this study, adopt or reject elearning as their new way of CE still needs to be confirmed, because e-learning does not suit every learner (Atack, 2003). Therefore, the main purposes of this study are to investigate the feasibility of elearning for establishing PHNs' continuing education and to further examine the reasons for adopting or rejecting e-learning as their alternative way of continuing education. Through analyses of reasons, we expect to increase the success of and reduce the barriers in developing e-learning.

\section{Method}

\section{Design}

This study employed a cross-sectional research design.

\section{Subjects}

A cluster random-sampling method was used to select sample. In this sampling method, first, all health centers were stratified into four strata: metropolitan city, county, township, and village. Then, health centers in each stratum were proportionally selected by a cluster random sampling; PHNs working at the selected health centers comprised the study sample. After the list of selected health centers was confirmed and oral approval obtained by municipal or county health bureaus and relevant health centers via telephone, we filed the official letter with the relevant centers for administrative recognition and support. Data were collected by mailing the questionnaire. Prior contact, continued follow up (weekly, three times), and provision of reward were used to increase the response rate in this study. Finally, of 280 recipients, 233 returned completed questionnaires, with a return rate of $83.2 \%$. Using a $\chi^{2}$ test, no significant differences were seen in PHN proportion in four levels between the sample and the population $\left(X^{2}=2.14\right.$, $P>0.05)$; i.e., the sample was representative of the population (Table 1 ).

\section{Instruments}

A self-administered questionnaire was used to collect data in this study. It included two main parts:

1. Independent variables: this category included age, education level, marital status, job position, types of health centers, and previous experience in web-based learning.

2. Dependent variables: this category included two parts, the feasibility of adopting e-learning as an alternative way of continuing education, and reasons for adopting or rejecting e-learning.

(1) Feasibility of adopting e-learning.

In this part, a four-point question was used to examine PHNs' perceived likelihood of adopting e-learning as their continuing education method in the future. "Definitely possible", "possible", "not possible", "definitely not possible" corresponded to points 4, 3, 2, and 1 , respectively. Scores greater than and equal to 3 meant affirmative possibility of adopting web-based learning; conversely, scores less than and equal to 2 meant negative possibility (i.e., rejection) of adopting e-learning.

(2) Reasons for adopting or rejecting e-learning. A structured questionnaire, called Reasons for Adopting or Rejecting E-learning Scale, was used to examine the reasons why PHNs adopt or reject e-learning. This scale was a 20 -item self-reporting scale. It included two separate dimensions: the reasons for adopting e-learning 
Table 1 Distribution of PHNs in different types of public health centers between population and sample

\begin{tabular}{|c|c|c|c|c|c|}
\hline \multirow[t]{2}{*}{ Types of health centers } & \multicolumn{2}{|c|}{ Population } & \multicolumn{2}{|c|}{ Sample } & \multirow[t]{2}{*}{$x^{2}$} \\
\hline & $N$ & $\%$ & $N$ & $\%$ & \\
\hline Metropolitan city & 335 & 13.54 & 42 & 18.03 & $\begin{array}{l}2.14 \\
(d f-3)\end{array}$ \\
\hline $\begin{array}{l}\text { County } \\
\text { Townships }\end{array}$ & $\begin{array}{l}434 \\
337\end{array}$ & $\begin{array}{l}17.54 \\
13.62\end{array}$ & $\begin{array}{l}36 \\
35\end{array}$ & $\begin{array}{l}15.45 \\
15.02\end{array}$ & \\
\hline Village & 1368 & 55.30 & 120 & 51.50 & \\
\hline Total & 2474 & 100.00 & 233 & 100.00 & \\
\hline
\end{tabular}

Note. Abbreviation: df, degree of freedom. $p>0.05$.

(11 items) and the reasons for rejecting elearning ( 9 items). For single items (i.e., reasons), a yes/no question was used to collect data; one point was given to "yes" answers, and zero points were given for "no" answers. Moreover, an open-ended question in each dimension was designed to collect extra reasons that were not listed in the questionnaire.

To evaluate questionnaire validity and reliability, six experts in the areas of distance education, health information, informational technology, nursing education, and community health nursing established the questionnaire's content validity. Three PHNs completed the face validity test. Regarding reliability of this study, the agreement of behavioural intention toward e-learning (a four-point question) was $90 \%$; an $r_{\mathrm{kr} 21}$ coefficient was used to examine internal consistency. For the Reason of Adopting or Rejecting E-learning Scale, an $r_{\mathrm{kr} 21}$ coefficient was 0.89 in pilot test with 30 PHNs indicating good reliability in internal consistency. For two dimensions of the Reason of Adopting or Rejecting E-learning Scale, "reasons for adopting e-learning" and "reasons for rejecting e-learning", $r_{\mathrm{kr} 21}$ coefficients were 0.88 and 0.78 , respectively.

\section{Ethical considerations}

This study was supported by the Taiwan government. Human rights and privacy of participants were protected. All subjects participated in this study based upon confidential and voluntary participation. Informed consents have been obtained from all participants in this study. Moreover, the study protocol, informed consent form, and questionnaire were reviewed and approved by the Institutional Review Board, Taipei Veterans General Hospital, to guarantee the subjects' rights and interests.

\section{Data analysis}

SPSS 10.0 (SPSS Inc., Chicago) for Windows was used to analyze data. Frequency and percentage were used to describe subjects' backgrounds as well as to investigate the feasibility of developing e-learning and to examine the reasons for adopting or rejecting e-learning for PHNs. $\chi^{2}$ test was used to examine the distribution differences of urbanization between the sample and population.

\section{Results}

\section{Basic data of the subjects}

The mean age of the participating subjects was 39.37 years $(S D=8.29)$. Over one half $(58.37 \%)$ had graduated from junior college; only $14.59 \%$ had a bachelors degree. Most subjects were married $(81.97 \%)$. About three-fourth $(72.10 \%)$ worked as registered nurses (RNs), $20.60 \%(n=43)$ worked as registered professional nurses (RPNs). Over one-half $(51.50 \%, n=120)$ of the subjects worked in village health centers. Participants' locations in metropolitan city, county, and township type were $18.03 \%, 15.45 \%$, and $15.02 \%$, respectively. Only $9.87 \%$ of the subjects had ever participated in an e-learning program (Tables 1 and 2).

\section{Feasibility of e-learning in PHNs' continuing education}

A total of 233 PHNs participated in this study. Most of the subjects $(88.84 \%, n=207)$ replied that it was "highly possible" or "possible" for them to take continuing education programs via e-learning in the future. However, twenty-six subjects $(11.16 \%)$ rejected e-learning as their alternative way of continuing education.

\section{Reasons for adopting or rejecting e-learning for PHNs}

Firstly, data were analysed as a whole. Among a total of 233 PHNs, more than one-half of subjects expressed that e-learning was beneficial for achieving lifelong learning (54.08\%),especially 
Table 2 Basic data of the subjects $(n=233)$

\begin{tabular}{lrr}
\hline Variables & $n$ & \multicolumn{1}{l}{$\%$} \\
\hline Personal factors & & \\
Age (Mean \pm SD = 39.37 $\pm 8.29 ;$ & & \\
$\quad$ range: $23-63$ years old) & 28 & 12.02 \\
$20-29$ & 83 & 35.62 \\
$30-39$ & 91 & 39.06 \\
$40-49$ & 31 & 13.30 \\
50 and above & & \\
Educational level & 63 & 27.04 \\
Vocational senior high school & 136 & 58.37 \\
Junior college & 34 & 14.59 \\
University and above & & \\
Marital status & 42 & 18.03 \\
Not married & 191 & 81.97 \\
Married & & \\
Job position & 168 & 72.10 \\
Registered nurse (RN) & 48 & 20.60 \\
Registered professional & & \\
nurse (RPN) & & \\
Head nurse & & \\
Previous experience in & & \\
$\quad$ web-based learning & & \\
Yes & & \\
No & & \\
\hline
\end{tabular}

because of allowing nurses to choose curriculum and content based on personal learning needs (52.79\%) (Table 3).

Then, according to the response of PHNs on feasibility, we divided participants into two groups: "adopting group" and "rejecting group".

In the adopting group $(n=207)$, who tended to adopt e-learning as the alternative way of continuing education, more than one-half of subjects expressed that e-learning was beneficial for them to achieve lifelong learning (60.87\%). Except achieving lifelong learning, the other five main reasons given for adopting e-learning as their alternative way of continuing education included: allowing them to select curriculum and content based on personal learning needs (59.42\%), broadening one's horizon and/or learning the newest knowledge (55.7\%), time-savings (55.07\%), based on job needs $(50.72 \%)$, and information diversity (50.24\%) (Table 3 ).

In contrast, the rejecting group $(n=26)$ indicated that poor computer competence $(84.62 \%)$, lack of adequate computer facilities (76.92\%), conflict or unmatching interests existing between job and education content $(69.23 \%)$, time limits $(69.23 \%)$, and heavy family duties and burdens $(65.38 \%)$ were the five main reasons PHNs rejected e-learning as their alternative way of continuing education (Table 4).

\section{Discussion and recommendation}

\section{The feasibility of e-learning in PHNs' continuing education}

Among a total of 233 subjects participating in this study, only 23 had ever previously enrolled in distance learning. Nearly nine-tenths $(88.84 \%)$ of subjects tended to adopt e-learning as their way of continuing education. This finding confirmed that e-learning (via the Internet) is a highly feasible way and worthy of development as a means of PHN continuing education. This finding is similar to those of Chapman (2000), and Kooker et al. (2000), which indicated that health professionals agree to the value of e-learning and tend to adopt e-learning as their way of continuing education, helping them to achieve the goal of lifelong learning.

Table 3 Main reasons for PHNs adopting e-learning programs

\begin{tabular}{|c|c|c|c|c|}
\hline Rank & Reasons for adoption & $n$ & $\begin{array}{l}\% \text { of the acceptors } \\
(n=207)\end{array}$ & $\begin{array}{l}\% \text { of the total } \\
(N=233)\end{array}$ \\
\hline 1 & Achieving lifelong learning & 126 & 60.87 & 54.08 \\
\hline 2 & Based on personal demand and interests & 123 & 59.42 & 52.79 \\
\hline 3 & Broaden one's horizon and/or learn the newest knowledge & 114 & 55.07 & 48.93 \\
\hline 3 & Time-saving in traffic for attending continuing education class & 114 & 55.07 & 48.93 \\
\hline 5 & Based on job needs: task-oriented & 104 & 50.72 & 45.06 \\
\hline 6 & Various information available (information diversity) & 103 & 50.24 & 44.64 \\
\hline 7 & Flexibility in time and space & 95 & 45.89 & 40.77 \\
\hline 8 & Self-regulatory learning & 93 & 44.93 & 39.91 \\
\hline 9 & Cost-effective & 83 & 40.10 & 35.62 \\
\hline 10 & $\begin{array}{l}\text { Opportunity to increase interaction with children: } \\
\text { accompanying with children learning from the internet }\end{array}$ & 72 & 34.78 & 32.67 \\
\hline 11 & Less impact on family life & 70 & 33.82 & 30.04 \\
\hline
\end{tabular}


Table 4 Main reasons for PHNs rejecting e-learning programs

\begin{tabular}{llrll}
\hline Rank & Reasons for rejection & $n$ & $\begin{array}{l}\% \text { of the rejectors } \\
(n=26)\end{array}$ & $\begin{array}{l}\% \text { of the total } \\
(N=233)\end{array}$ \\
\hline 1 & Poor computer competence & 20 & 84.62 & 9.44 \\
2 & No personal computer and internet access at home & 20 & 76.92 & 8.58 \\
3 & Conflict or unmatching interests existed between & 18 & 69.23 & 7.73 \\
& job and education content & & & \\
4 & Time limits & 18 & 69.23 & 7.73 \\
5 & Heavy family duties and burden; e.g., play caregiver role & 17 & 65.38 & 7.30 \\
& in taking care of children and parents & 10 & 38.46 & 4.29 \\
6 & Preference for face-to-face instruction & 8 & 30.77 & 3.43 \\
7 & Heavy economic burden & 4 & 15.38 & 1.72 \\
8 & Lack of motivation & 3 & 11.54 & 1.29 \\
9 & Heavy work burden and stress & 3 & 11.54 & 1.29 \\
9 & Low self-control, stop learning due to laziness & & & \\
\hline
\end{tabular}

\section{The reasons of adopting or rejecting e-learning for PHNs}

Most PHNs believe e-learning could fulfil their personal learning needs and job demands. How to integrate PHNs' personal needs and job demands is important, especially with the high challenge of designing curriculum and content. Soon et al. (2000) has proposed seven steps for developing elearning courses; the first step is analysis of the learning needs of learners. Learning needs assessment is critical before developing an e-learning course that is capable of fulfilling personal needs and job demands simultaneously. However, providing diverse learning programs might be more feasible for fulfilling various needs and demands. Content diversity, for example, has been one essential characteristic of e-learning (Yu and Yang, 2006). According to this finding, we emphasize three principles (diversity, individualization, and task-orientation) that should be considered in elearning curriculum development and content design. It is also necessary to increase search information ability and to provide clear e-learning information in order to help PHNs acquire desirable and effective e-learning programs.

PHNs in the adopting group believed that an elearning model could overcome the barriers of traditional face-to-face instruction models especially in addressing time and space barriers. This finding is similar to that of Cragg (1994), which indicates that an asynchronous e-learning style is more suitable than a synchronous e-learning style. However, in the rejecting group, $38.46 \%$ of the subjects were used to traditional face-to-face instruction. Thus, multi-channel learning patterns are still considered for PHN continuing education. Nearly one-half $(44 \%)$ of subjects in the adopting group (39.9\% of total subjects) believed e-learning to be a self-regula- tory learning style; $11.54 \%$ of subjects in the rejecting group expressed that low self-control is a barrier to adopting e-learning. These findings imply that for some PHNs, self-controlling ability might be one consideration for adopting or rejecting e-learning. For example, PHNs with high selfcontrol ability might tend to adopt e-learning; on the contrary, PHNs with low self-control ability then tend to reject e-learning. For individuals with less self-control, it is highly important to establish auser-friendly learning environment on the Internet in the beginning stages of development. Then, strategies of behaviour modification such as keeping learners' interest and attention, and increasing learners' achievement, could increase learners' confidence and self-controllability, thereby maintaining their participation and improving their learning effect.

The current study revealed that personal factors play important roles in either adopting or rejecting e-learning. In particular, poor computer competence was the main overall reason for rejecting elearning. Our finding is similar to those of Atack and Rankin (2002); Cragg (1994); Garland (1993); Kenny (2000); Leasure et al. (2000); and Yellen (1998). Although all public health centers in Taiwan are computerized (Department of Health, 2000), and PHNs regularly use specific work computer software (i.e., PHIS, Public Health Informatics System), these facts do not guarantee that PHNs have adequate computer competence for participating in e-learning programs. Basic computer competence improvement for PHNs is crucial for developing e-learning, because learners' adequate computer competence makes e-learning more acceptable and successful. Moreover, constructing a highly user-friendly platform and learning environments that reduce learners' fears would be useful in spreading the implementation of this new learning medium model. 
Regarding computer and internet access in households, the percentage of households owning a home computer in Taiwan in 2005 was 63.1\%; the percentage of households with an internet connection was 55.7\% (Directorate-General of Budget, R.O.C., 2006). Prevalence of personal computers and internet access at home in Taiwan are similar to developed countries. For example, the percentages of households owning a home computer in United States (United States Department of Labor, Bureau of Labor Statistics, 2005), United Kingdom (The Office for National Statistics, UK., 2005), Singapore (Infocomm Development Authority of Singapore, 2006), and Hong Kong-China (Census and Statistics Department, Hong Kong-China, 2004) were reported to be $54.7 \%, 62.0 \%, 74.0 \%, 71.1 \%$, respectively. The percentage of households with an internet connection in United States (United States Department of Labor, Bureau of Labor Statistics, 2005), United Kingdom (The Office for National Statistics, UK., 2005), Singapore (Infocomm Development Authority of Singapore, 2006), and Hong Kong-China (Census and Statistics Department, Hong Kong-China, 2004) were reported to be $61.8 \%, 53.0 \%, 66.0 \%, 64.9 \%$, respectively. Compared to developing countries, the percentages of households owning a home computer and with an internet connection are obviously higher than most developing countries (The World Bank, 2006).

For some PHNs, not having a home computer and being without an internet access are their stated barriers to adopting e-learning. For these PHNs, economic burden might impede them from having personal computers and internet access at home. Otherwise, on the whole, the percentages of penetration and usage of information technology in government offices and public enterprises in Taiwan is $100.0 \%$. As stated above, all public health centers in Taiwan are computerized (Directorate-General of Budget, R.O.C., 2006) and only a few PHNs did not have internet access at their worksite. Thus, there is a continuing need for government support to enable more people to access the internet. For example, government strategies for training PHNs in basic computer competence, will help ensure internet access at worksites, promising PHNs continuing education via e-learning and increasing incentives for learning (e.g., an official or academic certification). These steps will make development of e-learning as continuing education for PHNs both feasible and successful.

Administrative support is also critical in developing e-learning programs and in building supportive environments that motivate PHNs' participation in e-learning programs. However, for countries with lower percentages of computer use, such as certain developing countries (The World Bank, 2006), we still propose that the availability of computers and internet access is the basic element in developing effective e-learning programs.

Apart from computer hardware and software reasons, heavy workload, job stress and family duties all might impede PHNs' adoption of e-learning. These findings are similar to those of Atack and Rankin (2002), and Chapman (2000), which all indicate that time constraints due to work and family load still play important roles and make a negative impact on PHN continuing education. Therefore, in the future, education policy makers should consider how to increase PHNs' motivation and to improve PHNs' time-management skills so that they may be able to participate in e-learning and achieve improved educational outcomes.

\section{PHNs' previous e-learning experience}

One important statistic presented in this study is that only $9.87 \%$ of subjects had ever before participated in an e-learning program. Studies are lacking for comparison but we have compared this important data with percentages of personal computers and internet access in Taiwan. This obviously low rate of previous e-learning experience by PHNs participating in this study might imply that fewer PHNs have used the internet for professional development in past two decades, even though e-learning has been applied to nursing education ( $\mathrm{Yu}$ and Yang, 2006). Reinert and Fryback (1997) surveyed 353 schools of the American Nursing School Alliance and found that web-based courses have increased more than five-fold in the past 5 years. In the future, it is believed that the rate of e-learning will be maintained incrementally. Therefore, in order to produce a significant educational impact, it is important to conduct more studies to determine barriers to e-learning for nurses.

\section{Conclusion}

E-learning is a feasible and valuable learning model that is worth making generally available to PHNs, and indeed, to all health care professionals. From our reasons analyses findings, we have confirmed that asynchronous e-learning programs are a suitable method for PHN continuing education, especially for individuals with high self-control that allows PHNs to learn at remote locations according to their own needs and pace. Needs assessment is strongly recommended in the program preparation stage. Only by fulfilling PHNs' individual needs (personal and job), reducing learning barriers (time, space, computer 
competence, family and work load, etc.), increasing their motivation and self-controlling ability, can this new learning style be successful. Multi-channel learning patterns are still considered for inclusion in PHN continuing education because not all PHNs are accustomed to e-learning. Furthermore, regarding the effectiveness of e-learning programs, we suggest that further studies are required.

\section{Acknowledgement}

We acknowledge the National Science Council of Taiwan for funding this project (Project number: NSC 91-2314-B-010-065).

\section{References}

Atack, L., 2003. Becoming a web-based learner: registered nurses' experiences. Journal of Advanced Nursing 44 (3), 289-297.

Atack, L., Rankin, J., 2002. A descriptive study of registered nurses' experiences with web-based learning. Journal of Advanced Nursing 40 (4), 457-465.

Bernhardt, J.M., Runyan, C.W., Bou-Saada, I., Felter, E.M., 2003. Implementation and evaluation of a web-based continuing course in injury prevention and control. Health Promotion Practice 4 (2), 120-128.

Billings, D.M., Ward, J.W., Penton-Cooper, L., 2001. Distance learning in nursing. Seminars in Oncology Nursing 17 (1), 48-54.

Census and statistics department, the government of the Hong Kong special administrative region, 2004. Annual survey on information technology usage and penetration in the business sector for 2004. Available from: <http://www.censtatd.gov. hk/press_release/press_releases_on_statistics/index.jsp?sID= 4800\&sSUBID=-4800\&subject ID=\&charset $\mid \mathrm{D}=$ \&displayMode=D>.

Chapman, L., 2000. Distance learning for post-registered nursing: the facts. Nursing Standard 14 (18), 33-36.

Cook, G., Thynne, E., Weatherhead, E., Glenn, S., Mitchell, A., Bailey, P., 2004. Distance learning in post-qualifying nurse education. Nurse Education Today 24 (4), 269-276.

Cragg, C.E., 1994. Nurses' experiences of a post-RN course by computer mediated conferencing: friendly users. Computers in Nursing 12 (5), 221-226.

Department of Health, 2000. Republic of China Public Health Annual Report 2000. The Executive Yuan, Taipei.

DiMaria-Ghalili, R.A., Ostrow, L., Rodney, K., 2005. Webcasting: a new instructional technology in distance graduate nursing education. Journal of Nursing Education 44 (1), 11-18.

Directorate-General of Budget, Accounting and Statistics, Executive Yuan, R.O.C., 2006. Statistical Analysis of ROC Computer Resources Survey in 2005. Available from: <http://www. dgbas.gov.tw/public/Attachment/5102617403971.doc>.

Frith, K.H., Kee, C.C., 2003. The effect of communication on nursing student outcomes in a web-based course. Journal of Nursing Education 42 (8), 350-358.

Fullerton, J.T., Ingle, H.T., 2003. Evaluation strategies for midwifery education linked to digital media and distance delivery technology. Journal of Midwifery Women's Health 48 (6), 426-436.

Garland, M.R., 1993. Student perceptions of the situational, institutional, dispositional and epistemological barriers to persistence. Distance Education 14 (2), 181-198.

Gebbie, K.M., 1999. The public health workforce: key to public health infrastructure. American Journal of Public Health 89 (5), 660-661.

Infocomm Development Authority of Singapore, 2006. Annual Survey on Infocomm Usage in Households and by Individuals for 2005. Available from: <http://www.ida.gov.sg/idaweb/ manpower/infopage.jsp?infopagecategory=\&infopageid= 13760\&versionid $=7>$.

Kearns, L.E., Shoaf, J.R., Summey, M.B., 2004. Performance and satisfaction of second-degree BSN students in web-based and traditional course delivery environments. Journal of Nursing Education 43 (6), 280-284.

Kenny, A., 2000. Untangling the web. . .barriers and benefits for nurse education: an Australian perspective. Nurse Education Today 20, 381-388.

Kooker, B.M., Itano, J., Okimoto, H., 2000. Using focus groups to evaluate the impact of a masters in nursing distance education program. Journal of Nursing Education 39 (7), 329-332.

Leasure, A.E., Davis, L., Thievon, S.L., 2000. Comparison of student outcome and preferences in a traditional vs. world wide web-based baccalaureate nursing research course. Journal of Nursing Education 39 (4), 149-154.

Reinert, B.R., Fryback, P.B., 1997. Distance learning and nursing education. Journal of Nursing Education 36 (9), 421-427.

Soon, K.H., Sook, K., Jung, C.W., Kim, M., 2000. The effects of internet-based distance learning in nursing. Computers in Nursing 18 (1), 19-25.

The Office for National Statistics, UK, 2005. National Statistics: Consumer Durables. Available from: <http://www.statistics.gov.uk/cci/nugget_print.asp?ID=868>.

The World Bank, 2006. Information and Communications for Development (IC4D): Global Trends and Policies. ICT at a Glance Tables for 144 economics. Available from: <http:// web.worldbank.org/WBSITE/EXTERNAL/TOPICS/EXTINFORMATIONANDCOMMUNICATIONANDTECHNOLOGIES/0, contentMDK:20831214 pagePK:210058 piPK:210062 theSitePK: 282823,00.html>.

United States Department of Labor, Bureau of Labor Statistics, 2005. Computer and internet use at work in 2003. Available from: <http://www.bls.gov/news.release/pdf/ciuaw.pdf>.

Wills, C.E., Stommel, M., 2002. Graduate nursing students' precourse and postcourse perceptions and preferences concerning completely web-based courses. Journal of Nursing Education 41 (5), 193-201.

Yellen, R.E., 1998. Distance learning students: a comparison with traditional students. Journal Educational Technology Systems 26 (3), 215-224.

Yu, S., Chin, P.L., 1996. The roles of public health nurses in four different types of public health station in Taiwan. Public Health (Taiwan) 23 (1), 17-26.

Yu, S., Yang, K.F., 2006. Attitudes towards web-based distance learning among public health nurses in Taiwan: a questionnaire survey. International Journal of Nursing Studies 43 (6), 767-774. 